\title{
Emerging Relationship between Vitamin D and LL-37 in the Immune System's Response to Infection and Their Possible Role in Combating Sepsis
}

Nagwa M. Shawky ${ }^{1}$, Neveen G. EL-Antouny ${ }^{2}$, Noha K. Hassaan ${ }^{1}$, Alshymaa Ahmed Abdullah ${ }^{1}$

Departments of ${ }^{1}$ Clinical and Chemical Pathology, ${ }^{2}$ Internal Medicine, Zagazig University Hospital, Egypt

*Corresponding author: Noha Khaled Hassaan, EMail: noha.k.h@ hotmail.com

\begin{abstract}
Background: In patients who are critically unwell, sepsis could be one of major causes of mortality as well as many complications. Sepsis is the medical term describing dysfunctional body reaction to infection that results in dysfunctions in various body systems and hence severe organ failure could occur. A rise of 2 or more points in the sequential organ failure evaluation is an early indicator of organ dysfunction (SOFA score). Severe organ failure can occur if sepsis is left untreated. An infection-controlling effect of vitamin D has been demonstrated. With an increase in cathelicidin antimicrobial peptide (AMP) LL-37, human skin, blood plasma, monocytes, and macrophages are all affected. LL-37 and other AMPs, which operate directly against bacteria, regulate numerous innate and adaptive immunological functions. Objective: To make an overview of role of lack of vitamin D $(25(\mathrm{OH}) \mathrm{D})$ as well as AMP, LL-37, as risk factors for sepsis. Conclusion: Severe infections, as well as sepsis, have been linked to a deficiency of vitamin D. However, it is not yet clear what the underlying linkage and its clinical consequence is between sepsis and respiratory tract infections as well as critical illness.
\end{abstract}

Keywords: Sepsis, Vitamin D.

\section{INTRODUCTION}

Sepsis can progress from infection to septic shock, that might prime to mortality as well as multiple organ dysfunction syndrome, depending on the severity of the disease. When it comes to diagnosing and treating bacterial infections, a lot of changes have taken place since the early 1990s ${ }^{(1)}$. Septic patient definitions and clinical criteria have been updated by a task team of 19 professionals from critical care to infectious disease to pulmonary medicine in an effort to improve patient treatment. The sepsis-3 task force was formed as a result (2). Minor levels of vitamin D can be seen in cases with sepsis because of decrease in the vitamin D binding protein concentrations. Sepsis, additionally, vitamin D deficiency may have a role in the most catastrophic outcome of a severe illness ${ }^{(3)}$. It has not yet been clearly studied how vitamin D affects the development of severe sepsis in humans. Septic shock can occur due to deficiency of vitamin D supplementation. When it comes to the link between $25(\mathrm{OH}) \mathrm{D}$ and LL-37 in hospitalized patients, there are inconsistent results ${ }^{(4)}$. Vitamin D's function in general health, homeostasis of calcium as well as bone integrity has long been acknowledged. Researchers realizing the significance of this steroid hormone in the proper operation of numerous organ systems. More than 900 genes contain the vitamin D response element (VDRE), which is found in vitamin $\mathrm{D}$ receptors and the 25hydroxyvitamin D-1 $\alpha$-hydroxylase (1 $\alpha$-OHase). Systemic infections, cardiovascular illness, lung disease, and diabetes have been associated to a lack of vitamin D, based on current clinical as well as epidemiological investigations ${ }^{(5)}$.

Supplementation with Vitamin D and Immunity:

Aside from inflammation reduction and pathogen management, vitamin D is critical to the body's ability to fight off diseases through immunity reactions pathways. Staph aureus, Klebsiella pneumoniae, E. coli, and streptococcus pyogenes cultures were inhibited or destroyed in in vitro studies using 50,000 $90,000 \mathrm{IU} / \mathrm{ml}$ of active vitamin D (D3). Reductions in inflammation and virus replication were observed in tracheobronchial epithelial cells infected with respiratory syncytial virus (RSV) that were treated with $1,25(\mathrm{OH})_{2} \mathrm{D}$. Researchers found that injecting infected mammary tissue-derived monocytes led to higher levels of VDR and $1 \alpha$-OHase gene expression in a cow mastitis experimental model. With this study we can see how vitamin D-dependent immune responses can be activated in the face of local infection (6). Catheliciden (LL-37) is an antibacterial peptide, which have a broad range of activities. It was in the 1980 's that host defence peptides (AMPs) were first found. A wide variety of naturally occurring chemicals are produced by all multicellular organisms as a first line of defence against pathogens ${ }^{(7)}$. Antimicrobial activity of AMPs against bacteria, fungi, and viruses is demonstrated by their broad spectrum and high potency ${ }^{(8)}$.

Humans, animals, and plants all have an inherent immune system that relies on antimicrobial peptides ${ }^{(9,}$ 10). They are essential to the immune system because of their broad spectrum of activity against infections and cancerous cells. Defensin and cathelicidin are two types of antimicrobial peptides (AMPs) in mammals (11, 12).

Antimicrobial peptide LL-37 is able to fight a variety of infections. Many researches have showed that it has antibacterial and immunomodulatory characteristics. Recent research has focused on its ability to boost the body's natural defenses ${ }^{(\mathbf{1 2})}$. 


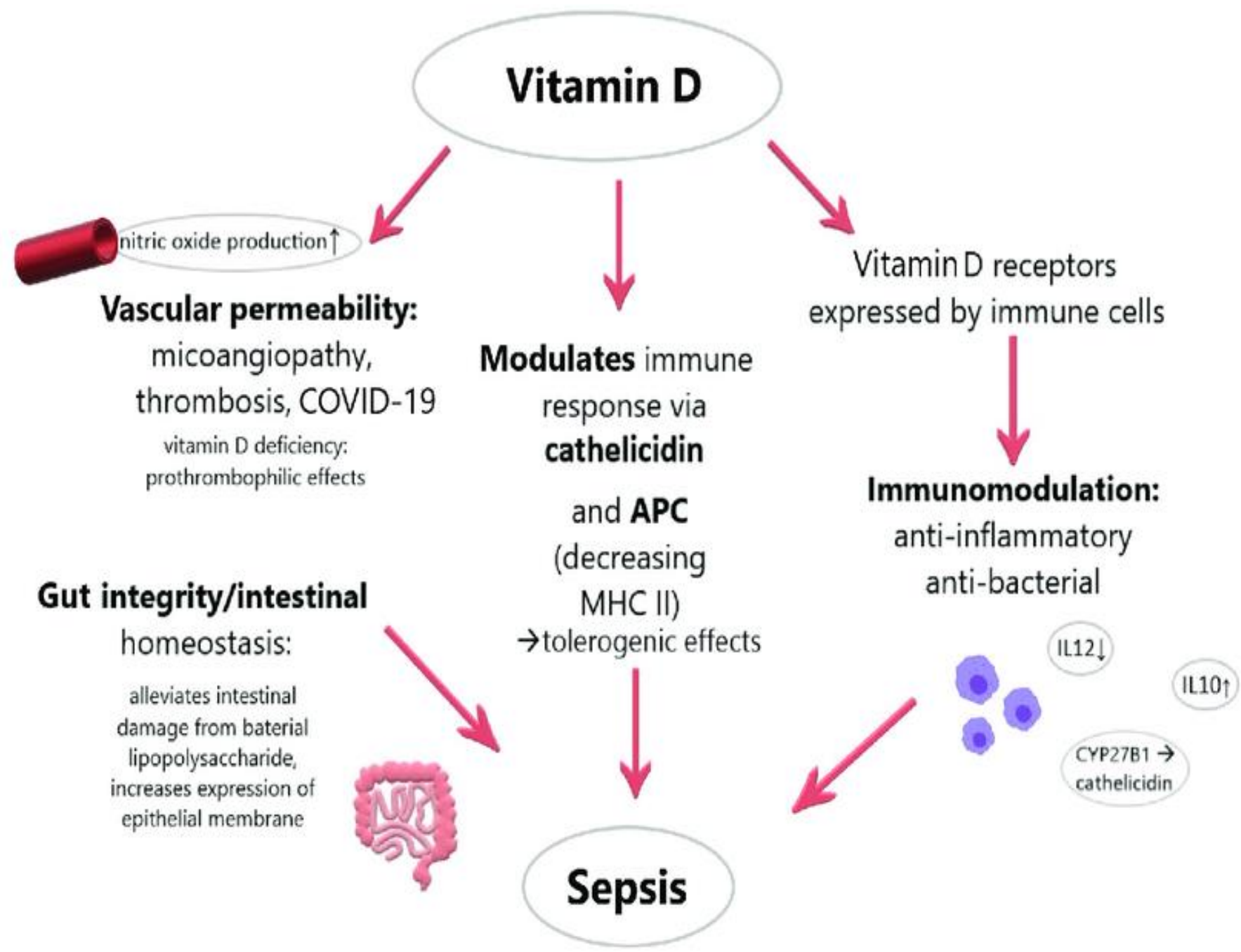

Figure (1): Vitamin D with sepsis: a quick review of the probable consequences ${ }^{(10)}$

As part of the immune response, peptides known as antimicrobials are also critical. Once within a phagosome, these peptides cause bacterial cell membranes to become more permeable, resulting in bacterial death. The monocyte pattern recognition receptors (PRRs) when bind to ligands of bacteria, they promote gene production of $1 \alpha$-OHase as well as the VDR when they are activated, according to research on vitamin D's participation in this process. In an autocrine mechanism, vitamin $\mathrm{D}$ response elements (VDRE) when being bound by $25(\mathrm{OH}) \mathrm{D}$, they activate AMPs genes like $\beta$ defensin 4A and hCAP 18. LL-37, the second molecule's active form, is then cleaved ${ }^{(13)}$.

LL-37's role in vitamin D-dependent innate immune systems has been extensively studied. Mucosal secretions and plasma contain it. Microbicidal effectiveness against significant human pathogens has been demonstrated for LL-37 in vitro involving, vancomycin-resistant entero-cocci, Staphylococcus aureus, Pseudomonas aeruginosa as well as Escherichia coli. Pseudomonas aeruginosa biofilm disintegration has been shown to be disrupted by this compound, as well as enhancing the ability of other immune cells to chemoattract to the infected areas ${ }^{(\mathbf{1 4})}$. $1,25(\mathrm{OH}) 2 \mathrm{D}$ was found to boost the production of LL-37 in human bronchial epithelial cells cultured with 1,25(OH)2D and to decrease the growth of airway pathogens Bordetella and Pseudomonas. The synthesis of LL-37 and bactericidal activity in human bladder cells was boosted when individuals were given oral vitamin $\mathrm{D}$ pretreatment in another investigation. AntiLL-37 antibodies were able to reverse this impact, underlining the importance of LL-37 in the vitamin Dmediated immunological response. Plasma levels of LL-37 and 25(OH)D were shown to be linked in intensive care unit patients, according to a study by Jeng and colleagues ${ }^{(14)}$. 


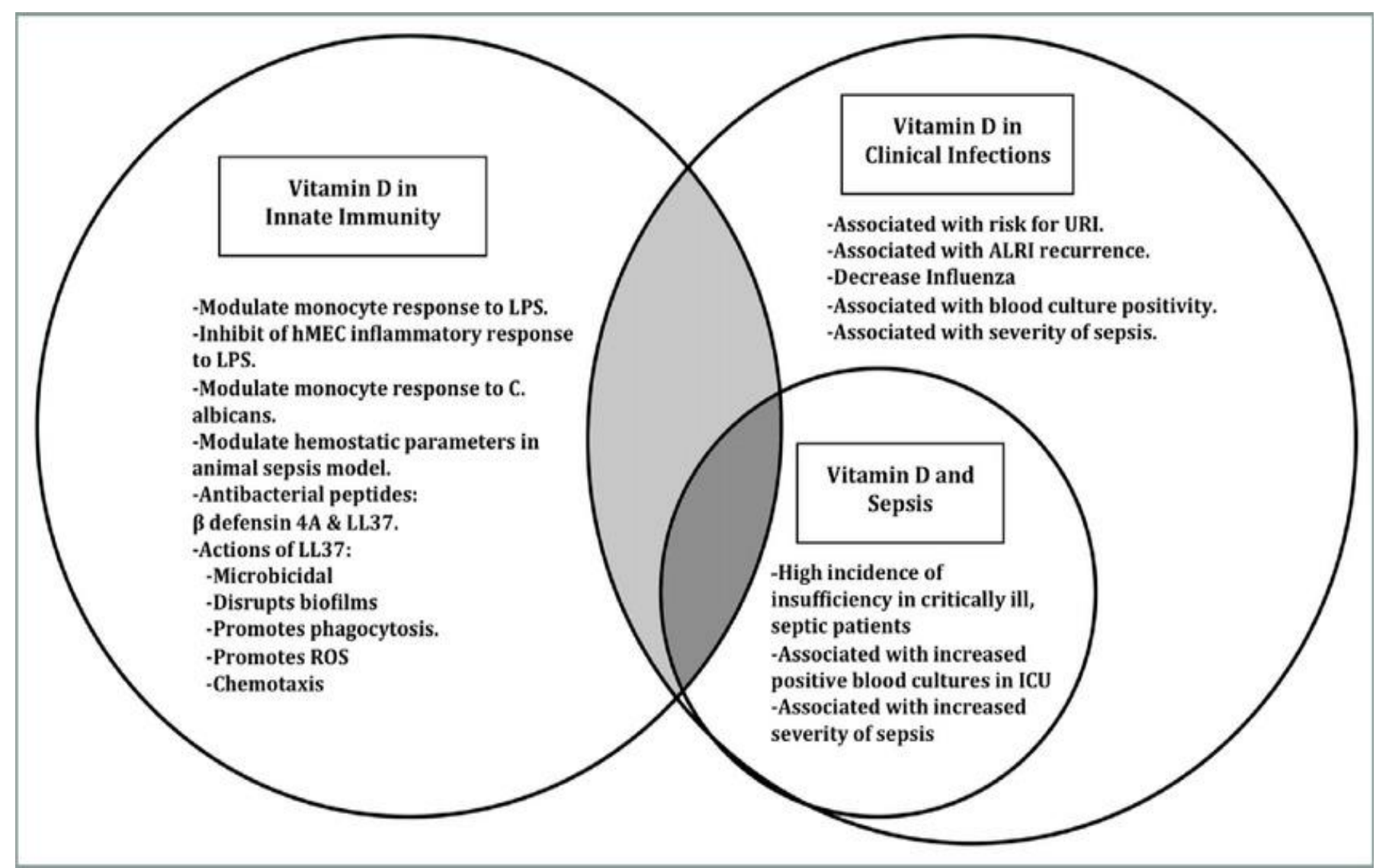

Figure (2): Mortality and illness a deficiency in vitamin D and the onset of sepsis ${ }^{(15)}$

\section{Cathelicidin (LL-37), Vitamin D and infection:}

Endogenous hCAP18 induction by vitamin D3 is widespread in a wide range of cell types ${ }^{(\mathbf{1 6}, \mathbf{1 7})}$, Vitamin $\mathrm{D}$ response elements (VDREs) in the cathelicidin antimicrobial peptide (CAMP) gene promoter sequence are linked to the VDR, a transcription factor from the steroid/hormone receptor family ${ }^{(\mathbf{1 8})}$. Primatespecific regulation is a sort of evolutionarily-derived regulation ${ }^{(16)}$.

Signaling cascades triggered by mycobacteria result in the up-regulation of expression for both VDR and hydrolase CYP27B1 that changes inactive provitamin $\mathrm{D}$ to the active form, therefore enhancing vitamin D efficacy ${ }^{(19)}$. VDR activation rather than TLR activation causes an increase in HCAP18 expression. HCAP18 levels in the blood have been demonstrated to be affected by vitamin $\mathrm{D}$ for example, keratinocytes in the airways or skin's epithelial surfaces play an important part in improving antimicrobial defense ${ }^{(\mathbf{1 7}, \mathbf{2 0})}$.

Immunomodulatory cytokines, which are released as a result of TLR activation, are made more readily available to the body. There has been evidence that one of the most important cytokines, IL-15, is essential for TLR2/1 activation of cathelicidin and adequate in macrophages, vitamin $\mathrm{D}$ can be activated. What exactly IL-15 does is still unknown, however it may be able to stimulate the vitamin D pathway in necrotizing cells as well as macrophages, hence enhancing innate immune response ${ }^{(\mathbf{2 1})}$.
Humans only have one antimicrobial peptide that is controlled by vitamin D, and LL-37 is the sole one ${ }^{(11)}$. Nielsen and colleagues ${ }^{(22)}$ discovered that urinary tract infection risk could be influenced by LL37 concentrations. Septic shock was revealed to regulate LL-37 through a complicated route by Barbeiro and colleagues ${ }^{(23)}$.

Deficiency in vitamin D has been linked to an increased risk of a wide range of infections. In epidemiological research, vitamin D deficiency has been associated to an increased risk of respiratory tract infections ${ }^{(24)}$. Epidemic influenza may be caused by a lack of vitamin D, according to one theory ${ }^{(25)}$. In the first trimester of pregnancy, it has been found that bacterial vaginosis is associated with maternal vitamin D deficiency (26). HIV-infected patients have been found to have low amounts of vitamin $\mathrm{D}$, and this may have an effect on how quickly the disease progresses (27). According to researches of epidemiology for respiratory illness, the vitamin D path is triggered in cells of lung through TLR3 signalling, leading to the activation of the CAMP gene in response to RSV infection ${ }^{(6)}$. Vitamin D deficiency may hinder this response by lung cells.

Transient production of the endogenous cathelicidin (hCAP18/LL-37) protein, it was found to be 10 times more effective than the use of synthetic antimicrobial peptide injections to prevent the spread of bacteria on burns ( $2^{\text {nd }}$ degree types) in rats, according to a recent study ${ }^{(28)}$. As for Shigella-infected rabbits, butyrate treatment led to a better outcome ${ }^{(29)}$. 
Aside from its antibacterial properties, severe sepsis can be prevented by hCAP18. Synthetic hCAP18/LL37 may be helpful, but it has been predicted that therapies that augment endogenous hCAP18 levels may be particularly beneficial in the treatment of infections, as butyrate- or adenoviral vector-induced increases in endogenous hCAP18 led significantly improved outcomes ${ }^{(30)}$.

\section{Conclusion:}

When sepsis develops, vitamin D has been found to play a significant role in both the local and systemic inflammatory pathways. There has been links between sepsis and respiratory tract infections as well as critical illness, but the causative linkage and its clinical influence have not yet been clearly confirmed by clinical researchers. Vitamin D is inexpensive and safe, and the beneficial roles in sepsis outcomes can be applied on a broad scale, hence additional investigation is advised.

Financial support and sponsorship: Nil. Conflict of interest: Nil.

\section{REFERENCES}

1. Neviere R, Manaker S, Sexton D et al. (2018): Sepsis syndromes in adults: Epidemiology, definitions, clinical presentation, diagnosis, and prognosis. https://www.uptodate.com/contents/sepsis-syndromes-inadults-epidemiology-definitions-clinical-presentationdiagnosis-and-prognosis

2. Ho V, Kaafarani H, Rattan R et al. (2020): Sepsis 2019: What surgeons need to know. Surgical Infections, 21: 195-204.

3. Møller S, Laigaard F, Olgaard K et al. (2007): Effect of 1,25-dihydroxy-vitamin D3 in experimental sepsis. Int J Med Sci., 4:190-195.

4. Li Y, Ding S (2020): Serum 25-Hydroxyvitamin D and the risk of mortality in adult patients with Sepsis: a metaanalysis. BMC Infect Dis., 20: 189: 1-10.

5. Kempker J, Han J, Tangpricha V et al. (2012): Vitamin $\mathrm{D}$ and sepsis: An emerging relationship. Dermatoendocrinology, 4(2): 101-108.

6. Hansdottir S, Monick M, Hinde S et al. (2008): Respiratory epithelial cells convert inactive vitamin D to its active form: potential effects on host defense. $J$ Immunol, 181:7090-7099.

7. Zhang L, Gallo $\mathbf{R}$ (2016): Antimicrobial peptides. Current Biology, 26: 14-19.

8. Bahar A, Ren D (2013): Antimicrobial peptides. Pharmaceuticals (Basel, Switzerland), 6: 15431575 .

9. Kosciuczuk E, Lisowski P, Jarczak J et al. (2012): Cathelicidins family of antimicrobial peptides. A review. Mol Biol Rep., 39:10957-10970.

10. Starr C, Maderdrut J, He J et al. (2018): Pituitary adenylate cyclase-activating polypeptide is a potent broad-spectrum antimicrobial peptide: structure-activity relationships. Peptides, 104:35-40.

11. Vandamme D, Landuyt B, Luyten W et al. (2012): A comprehensive summary of LL-37, the factotum human cathelicidin peptide. Cell Immunol., 280: 22-35.

12. Yang B, Good D, Mosaiab T et al. (2020): Significance of LL-37 on immunomodulation and disease outcome. BioMed Research International, 20: 1-16.

13. Hewison M (2011): Antibacterial effects of vitamin D. Nat Rev Endocrinol., 7:337-45.

14. Jeng L, Yamshchikov A, Judd S et al. (2009): Alterations in vitamin D status and anti-microbial peptide levels in patients in the intensive care unit with sepsis. Journal of Translational Medicine, 7: 1-9.

15. Kempker J, Tangpricha V, Ziegler T et al. (2012): Vitamin D in sepsis: from basic science to clinical impact. Critical Care, 16: 1-6.

16. Gombart A (2009): The vitamin D-antimicrobial peptide pathway and its role in protection against infection. Future Microbiol., 4: 1151-1165.

17. Dixon B, Barker T, McKinnon T et al. (2012): Positive correlation between circulating cathelicidin antimicrobial peptide (hCAP18/LL-37) and 25-hydroxyvitamin D levels in healthy adults. BMC Res Notes, 5: 1-5.

18. Gombart A, Borregaard N, Koeffler H (2005): Human cathelicidin antimicrobial peptide (CAMP) gene is a direct target of the vitamin $\mathrm{D}$ receptor and is strongly upregulated in myeloid cells by 1,25-dihydroxyvitamin D3. FASEB J., 19:1067-1077.

19. Liu P, Stenger $S$, Li H et al. (2006): Toll-like receptor triggering of a vitamin D-mediated human antimicrobial response. Science, 311: 1770-1773.

20. Schauber J, Gallo R (2008): The vitamin D pathway: a new target for control of the skin's immune response? Exp Dermatol., 17: 633-639.

21. Krutzik S, Hewison M, Liu P et al. (2008): IL-15 links TLR2/1-induced macrophage differentiation to the vitamin D-dependent antimicrobial pathway. J Immunol., 181: 7115-7120.

22. Nielsen K, Dynesen P, Larsen $P$ et al. (2014): Role of urinary cathelicidin LL-37 and human $\beta$-defensin 1 in uncomplicated Escherichia coli urinary tract infections. Infect Immun., 82: 1572- 1578.

23. Barbeiro D, Barbeiro H, Zampieri F et al. (2013): Cathelicidin LL-37 bloodstream surveillance is down regulated during septic shock. Microbes Infect., 15: 342346.

24. Walker V, Modlin R (2009): The vitamin D connection to pediatric infections and immune function. Pediatr Res., 6:106-113.

25. Cannell J, Vieth $\mathbf{R}$, Umhau $\mathbf{J}$ et al. (2006): Epidemic influenza and vitamin D. Epidemiol Infect., 134:1129 1140.

26. Bodnar L, Krohn M, Simhan H (2009): Maternal vitamin D deficiency is associated with bacterial vaginosis in the first trimester of pregnancy. J Nutr., 139:1157-1161.

27. Villamor E (2006): A potential role for vitamin D on HIV infection? Nutr Rev., 64:226-233.

28. Jacobsen F, Mittler D, Hirsch T et al. (2005): Transient cutaneous adenoviral gene therapy with human host defense peptide hCAP-18/LL-37 is effective for the treatment of burn wound infections. Gene Ther., 12:1494-1502.

29. Zasloff $M$ (2006): Inducing endogenous antimicrobial peptides to battle infections. Proc Natl Acad Sci USA., 103: 8913-8914.

30. Raqib R, Sarker P, Bergman $P$ et al. (2006): Improved outcome in shigellosis associated with butyrate induction of an endogenous peptide antibiotic. Proc Natl Acad Sci USA., 103: 9178-9183. 